$54^{\text {ème }}$ Congrès de la SFMBCB, 03017 (2011)

DOI: $10.1051 / \mathrm{sfmbcb} / 20115403017$

(C) Owned by the authors, published by EDP Sciences, 2011

\title{
Impression d’éléments biologiques par LIFT : intérêt en ingénierie tissulaire osseuse
}

\author{
Catros $\mathrm{S}^{1,2}$, Fricain $\mathrm{JC}^{1,2}$, Guillemot $\mathrm{F}^{2}$ \\ ${ }^{1}$ Pôle Odontologie et Santé buccale, Hôpital Pellegrin, Faculté de Chirurgie Dentaire, Université \\ Victor Segalen Bordeaux 2, France \\ ${ }^{2}$ Inserm U577, Biomatériaux et Réparation tissulaire, Université Victor Segalen Bordeaux 2, \\ France \\ sylvaincatros@hotmail.com
}

Introduction: L'ingénierie tissulaire utilise des méthodes issues des sciences du vivant et de l'ingénieur appliquées à la régénération de tissus vivants (Langer 1999). Cette approche peut être utilisée pour la reconstruction osseuse en utilisant des échafaudages dégradables, colonisés par des cellules endothéliales et osseuses (Morishita 2006). Cependant, cette méthode ne permet pas d'organiser spatialement chaque élément du tissu reconstruit. Face à ces limites, plusieurs méthodes (jet d'encre, micropipettes, laser) ont été développées afin d'organiser dans l'espace les différents constituants du tissu (Doraiswamy 2007, Mironov 2008). L'impression d'éléments biologiques par laser (Laser Induced Forward Transfer: LIFT) a été utilisée dans ce travail afin d'imprimer des ostéoblastes et de l'hydroxyapatite nano-cristalline avec une résolution spatiale contrôlée. Plus globalement, l'objectif était d'étudier l'intérêt de cette méthode pour produire des matériaux de reconstruction osseuse.

Matériel et méthodes: Une solution d'hydroxyapatite nano-cristalline a été préparée par précipitation (Saeri 2003), puis a été caractérisée par microscopie électronique à balayage (MEB) et à transmission (MET), par diffraction $X$ et par spectroscopie infra-rouge (FTIR). Ensuite, on a ajouté $30 \%$ de glycérol à la solution afin d'améliorer sa viscosité. Par ailleurs, des cellules ostéoblastiques de lignée murine (MG63) ont été cultivées, puis suspendues dans du sérum de veau fœtal (20 millions.ml $\left.{ }^{-1}\right)$. Ces deux solutions ont été utilisées pour réaliser des impressions en deux ou trois dimensions grâce à la méthode LIFT. Un laser impulsionnel infra-rouge (Nd:YAG 1064 $\mathrm{nm}, 30 \mathrm{~ns}$ ), piloté par des miroirs galvanométriques, a été focalisé sur une lame de verre recouverte d'une couche absorbante de titane de $30 \mathrm{~nm}$. Cette « lame donneuse » était recouverte d'environ 20 $\mu 1$ de la solution d'hydroxyapatite ou de cellules sur sa face inférieure, formant ainsi un film de 50 $\mu \mathrm{m}$ environ sur la couche de titane. Une autre lame de verre (lame réceptrice) était placée à $300 \mu \mathrm{m}$ de la lame donneuse, sur laquelle différents motifs étaient imprimés. Plusieurs paramètres, contrôlés grâce à une interface informatique dédiée (Station LASIT, Novalase, Canéjan), ont été testés pour les impressions; ensuite, les résultats ont été caractérisés par microscopie optique, MEB et profilométrie optique.

Résultats : Des spectres caractéristiques de l'hydroxyapatite ont été retrouvés en FTIR et en DRX, et des cristaux de $50 \mathrm{~nm}$ environ ont été observés en MET. Concernant les projections, la résolution optimale a été obtenue avec une fluence de $100 \mathrm{~mJ} . \mathrm{cm}^{-2}$. Ainsi, en variant la vitesse des scanners, on a pu imprimer des gouttes de 50 à $80 \mu \mathrm{m}$ de diamètre et de $20 \mu \mathrm{m}$ de hauteur ou bien des lignes continues ou des surfaces plus larges. Dans un second temps, les cellules ont été imprimées sur la couche précédente avec la même résolution et plusieurs couches ont ainsi pu être superposées.

Discussion: Ces premiers résultats montrent que deux des constituants essentiels de l'os (hydroxyapatite et ostéoblastes) peuvent être disposés précisément en 3 dimensions par la méthode d'impression d'éléments biologiques par laser. La prochaine étape de ce travail aura pour objectif d'améliorer les propriétés mécaniques des constructions tridimensionnelles en utilisant un hydrogel ou du plasma riche en plaquettes. Par ailleurs, une étude préliminaire est en cours avec pour objectif de reconstruire in vivo, par la même méthode LIFT, des défauts osseux critiques pratiqués sur la calvaria de souris grâce à la solution d'hydroxyapatite précédemment décrite.

This is an Open Access article distributed under the terms of the Creative Commons Attribution-Noncommercial License 3.0, which permits unrestricted use, distribution, and reproduction in any noncommercial medium, provided the original work is properly cited. 